\title{
DIAGRAMA DE MÍNIMO CONSUMO DE CARBONO APLICAÇÃO AO ALTO FORNO*
}

\author{
Enrique Roy Dionisio Calderon ${ }^{1}$ \\ José Carlos D'Abreu² \\ Helio Marques Kohler ${ }^{3}$
}

\section{Resumo}

Neste trabalho é apresentada a concepção de um modelo com objetivo de gerar a superfície de mínimo consumo de redutor (carbono) para a produção de ferro primário. No seu desenvolvimento foram consideradas as condições de equilíbrio das reações de redução e os balanços de massa e de energia. Sua aplicação à redução em Altos Fornos é exemplificada para situações reais, baseada no banco de dados operacionais de uma usina siderúrgica. Foi possível, a partir de planos de corte nestas superfícies, gerar diagramas binários do tipo CR-RD (Consumo de Redutor-Redução Direta), passíveis de serem obtidos "on-line" com o processo. Dentre os resultados obtidos, foi possível identificar, confirmar e prever, através da análise de casos reais, algumas inviabilidades termodinâmicas importantes do forno, conferindo desta forma maior segurança operacional ao processo.

Palavras-chave: Alto forno; Carbon "rate"; Redução direta; Balanço de massa; Balanço de energia.

\section{DIAGRAM FOR MINIMUM CARBON CONSUMPTION. APPLICATION TO THE BLAST FURNACE}

\section{Abstract}

In this paper a model is presented with the objective to generate the surface of minimum consumption of reductant (carbon) for ironmaking processes. In developing the model the equilibrium conditions of the reduction and the mass and energy balances were considered. Its application to the blast furnaces is exemplified for real operational conditions, which were databases duly extracted from an integrated steelworks. It was also possible, from cutting plans on these surfaces, to obtain binary diagrams of RC-DR type (Reductant Consumption-Direct Reduction), able to be generated on-line with the process. Among the results, one could identify, confirm and predict through analysis of real case studies, important thermodynamic unfeasibility of the furnace, which allowed to provide greater operational safety to the process.

Keywords: Blast furnace, Carbon rate; Direct reduction; Mass balance; Energy balance.

1 M Sc, Eng. Químico. Doutorando em Engenharia Metalúrgica e Materiais do Departamento de Engenharia de Materiais (DEMa) da Pontifícia Universidade Católica do Rio de Janeiro (PUCRio), Rio de Janeiro, RJ, Brasil.

2 D Sc, Professor Emérito, DEMa, PUC-Rio, Rio de Janeiro, RJ, Brasil.

3 Ph D, Eng. Metalúrgico pesquisador do Grupo de Siderurgia, DEMa, PUC-Rio, Rio de Janeiro, RJ, Brasil.

* Contribuição técnica ao $44^{\circ}$ Seminário de Redução de Minério de Ferro e Matérias-primas, 15ํㅗ Simpósio Brasileiro de Minério de Ferro e 2º Simpósio Brasileiro de Aglomeração de Minério de Ferro, 15 a 18 de setembro de 2014, Belo Horizonte, MG, Brasil. 


\section{INTRODUÇÃO}

Em 2013 a produção mundial de aço aumentou cerca de 3,5\%, alcançando mais de 1,6 bilhões de toneladas. No Brasil, a produção de 34,2 milhões de ton de aço, em 2013, foi devida $77 \%$ ao alto forno, reator que ainda se constitui na melhor opção para obtenção de ferro gusa [11]. As circunstâncias operacionais nos dias de hoje, muito afetadas por custos mais altos, matérias primas com qualidades inferiores, crise financeira internacional persistente e uma capacidade ociosa elevada, tornam cada vez mais importante o desenvolvimento de métodos e procedimentos que ajudem a enfrentar os principais fatores influentes no custo do ferro gusa $[1,4,5]$.

O carbono é o principal insumo usado como redutor dos óxidos de ferro no alto forno. Como seu emprego, via coque ou carvão vegetal, sempre representou significativa percentagem no custo do gusa, manter seu consumo em níveis ótimos sempre constituiu meta importante dos operadores. Portanto, é fundamental conhecer os limites que satisfaçam as restrições termodinâmicas e assegurem o provimento da quantidade de carbono para a estequiometria e a demanda de energia das reações de redução, nas condições específicas de cada processo $[4,7]$.

Neste contexto, é apresentado o desenvolvimento de uma superfície de mínimo consumo de carbono-SMCC, com a finalidade de contribuir na tomada de decisão e análise da condução dos processos de produção de ferro primário. Esta superfície foi concebida atendendo essencialmente os equilíbrios das reações e os balanços de massa e de energia, de forma a satisfazer a demanda total de carbono para a redução. No presente trabalho é apresentada sua aplicação ao caso de um alto forno a coque [2,3,6-10].

\section{DESENVOLVIMENTO}

Foram realizados os equacionamentos dos balanços estagiados de massa, energia, e global de um alto forno com base nos algoritmos e diagramas clássicos de Michard, Rist e as regiões de preparação e elaboração, onde ocorrem as trocas térmicas e de oxigênio.

Definiu-se como redução indireta, $\mathrm{Rl}$, a reação dos óxidos ferrosos com o gás redutor $\mathrm{CO}$, gerando $\mathrm{CO}_{2}$, e como redução direta, $\mathrm{RD}$, a reação global constituída pela redução indireta e a reação de Boudouard.

O equacionamento do balanço global de massa forneceu a equação 1 :

$$
X+Y+V=(1-R D)(n-G)\left(1+\frac{a-d}{b+d}\right)
$$

Onde $\mathrm{X}$ : átomos de carbono da Redução Direta;

Y: átomos de carbono existentes no gás $\mathrm{CO}$ produzido pela reação de Boudouard;

V: átomos de carbono existentes no gás $\mathrm{CO}$ produzido pela redução da água;

RD: porcentagem de redução direta;

$\mathrm{n}$ : átomos de oxigênio presentes nos óxidos de ferro, a serem reduzidos;

G: átomos de oxigênio presentes nos óxidos não ferrosos, a serem reduzidos;

a: $\quad$ átomos de oxigênio presentes no $\mathrm{CO}$ do gás de topo;

b: átomos de oxigênio presentes no gás $\mathrm{CO}_{2}$ do gás de topo;

d: átomos de oxigênio presentes no $\mathrm{H}_{2} \mathrm{O}$ do gás de topo.

* Contribuição técnica ao $44^{\circ}$ Seminário de Redução de Minério de Ferro e Matérias-primas, 15은 Simpósio Brasileiro de Minério de Ferro e $2^{\circ}$ Simpósio Brasileiro de Aglomeração de Minério de Ferro, 15 a 18 de setembro de 2014, Belo Horizonte, MG, Brasil. 
$\mathrm{Na}$ zona de elaboração determinou-se o carbono total necessário para satisfazer o equilíbrio (carbono consumido pelo sôpro; pela redução do $\mathrm{SiO}$ em $\mathrm{Si}$; pela redução do $\left.\mathrm{H}_{2} \mathrm{O}\right)$.

$$
C E Q=\frac{1}{K_{C o}}\left[1.05+\omega+G-\left(X+\frac{K_{H} \times Z}{K_{H}+1}\right)\right]
$$

Onde CEQ: carbono necessário para satisfazer o equilíbrio .

Kco: constante de equilíbrio da redução de $\mathrm{FeO}$ pelo $\mathrm{CO}$

$\omega: \quad$ fator Omega de Rist

$\mathrm{K}_{\mathrm{H}}: \quad$ constante de equilíbrio da redução do $\mathrm{FeO}$ pelo $\mathrm{H}_{2}$

Z: $\quad$ átomos de hidrogênio produzidos pela redução da água

A seguir, determinou-se o carbono necessário para satisfazer as demandas térmicas do balanço de energia das regiões de elaboração e de combustão::

$$
C B T=\frac{C G E}{C G S}
$$

Onde, CBT: quantidade de carbono necessário para satisfazer a demanda térmica, por átomo de ferro;

CGE: calor demandado para satisfazer as necessidades térmicas, por átomo de ferro

CGS: calor fornecido pela combustão do átomo de carbono.

O equacionamento para a geração da superfície de mínimo consumo de carbono foi realizado levando-se em conta que, em altas taxas de Redução Direta, o equilíbrio da redução da wüstita via intermediário gasoso define o consumo de carbono. Entretanto, sendo a Redução Direta endotérmica, ela também demandará carbono para satisfazer esses requisitos térmicos. Por outro lado, quando as taxas de Redução Indireta forem altas, existirá diminuição daquela demanda térmica e, portanto, menos carbono para aquele fim. Porém, devido o equilíbrio estequiométrico da redução indireta, haverá aumento na demanda de CO.

O equacionamento para a geração da superfície de mínimo consumo de carbono como função das variáveis $R$ e $R D$, está representado pelas equações (4) e(5):

Quando: CEQ>CBT

$C C_{e q}=C E Q \times K_{C o} \times\left[\frac{R}{K_{H}}+1\right]+C_{G U S A}$

Ou, quando: $\mathrm{CBT}>\mathrm{CEQ}$

$$
C C_{e n}=\left(\frac{R}{K_{H}}+1\right) \times\left[\frac{-10^{3}(C B T \times C G S-\theta)(A-G)-(R D \times A-G) \Delta H^{\prime \prime}}{\Delta H^{\prime \prime}}\right]+C_{G u s a}
$$

Onde CCeq: Carbono mínimo consumido no processo, considerando o equilíbrio;

CCen: Carbono mínimo consumido, considerando o balanço térmico;

CGusa: Carbono contido no gusa

R: $\quad$ Relação entre $\mathrm{CO}, \mathrm{CO}_{2}$ e $\mathrm{H}_{2}, \mathrm{H}_{2} \mathrm{O}$, no gás de topo;

A: fator igual a $1,05+\omega+\mathrm{G}$

$\theta$ : função contendo perdas térmicas, calor contido na gusa e na escória, e as entalpias das reduções dos óxidos não ferrosos e reduções pelo $\mathrm{H}_{2}$.

$\Delta \mathrm{H}_{\mathrm{RD}}:$ Entalpia para a Redução Direta.

$\Delta H^{\prime \prime} \mathrm{RI}$ Entalpia para a Redução Indireta.

* Contribuição técnica ao 44 Seminário de Redução de Minério de Ferro e Matérias-primas, 15은 Simpósio Brasileiro de Minério de Ferro e $2^{\circ}$ Simpósio Brasileiro de Aglomeração de Minério de Ferro, 15 a 18 de setembro de 2014, Belo Horizonte, MG, Brasil. 
A consideração das duas superfícies de consumo de carbono, uma devido ao equilíbrio, expressa pela eq. (4), e outra devido ao balanço térmico, representada pela eq. (5), conduz a uma superfície resultante, a qual satisfará sempre ao maior valor entre $\mathrm{CC}_{\text {eq }}$ e $\mathrm{CC}_{e n}$, de acordo com a lógica da eq. (6):

$$
\mathbf{C C}_{\text {eff }}=\left\{\mathbf{C C}_{\mathrm{eq}}, \mathrm{CC}_{\mathrm{en}}\right\}_{\text {supremo } \forall \mathbf{R}, \mathbf{R D}}
$$

Onde $\mathrm{CC}_{\text {eff }}$ : é o Carbono mínimo consumido resultante, expresso como

Consumo de Carbono efetivo(at-C/at-Fe).

A figura 1 ilustra uma superfície de mínimo consumo de carbono.

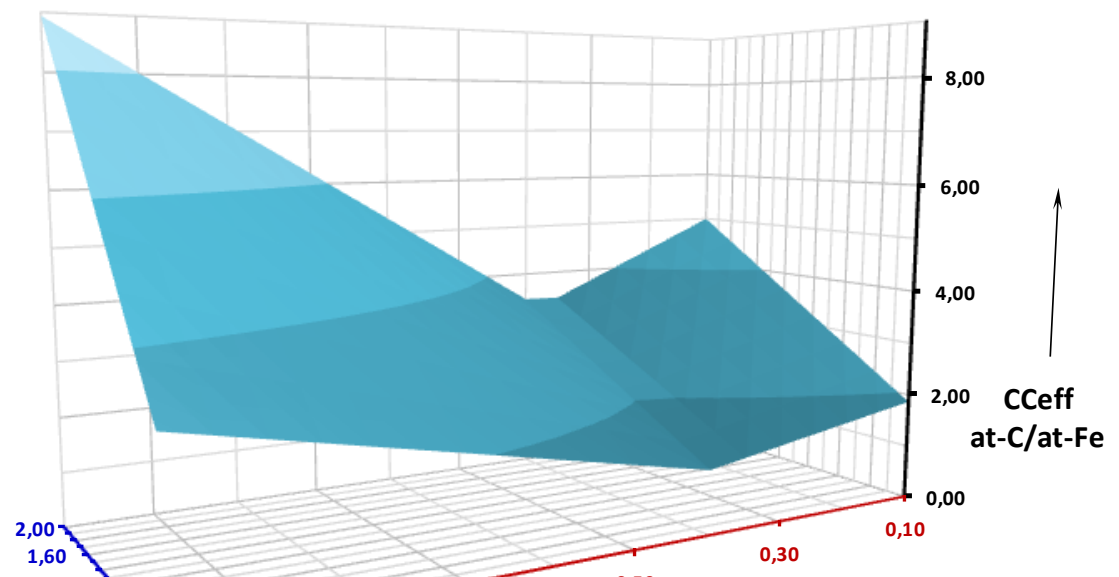

Figura 1. Diàgramna GCeff $\times \mathrm{R} \times \mathrm{RD}$, da s.,yperfície de mínime consumo de carbono, onde os pontos acima da superfície represengtam condições viáveis termodinamicamente, e os pontos abaixo condições inviáveis.

A figura 2 mostra um corte 2D, do tipo CR-RD (Consumo de Redutor-Redução Direta), da superfície da figura 1 , gerado por um plano definido por $\mathrm{R}$ constante. Nesta figura, o consumo efetivo de carbono ( $\mathrm{CC}_{\text {eff }}$ ) foi transformado em "Coke Rate", obedecendo à eq. 7 abaixo (9), afim de atender objetivos mais aplicados.

$$
C R=214,869 \times C C_{e f f} \times\left(\frac{\% F e}{C_{f i x o}}\right)+L A P O
$$

Onde LAPO: representa as quantidades de pó de coletor e lama, em kg de coque/tonelada de gusa.

* Contribuição técnica ao $44^{\circ}$ Seminário de Redução de Minério de Ferro e Matérias-primas, 15ํ Simpósio Brasileiro de Minério de Ferro e 2 Simpósio Brasileiro de Aglomeração de Minério de Ferro, 15 a 18 de setembro de 2014, Belo Horizonte, MG, Brasil. 

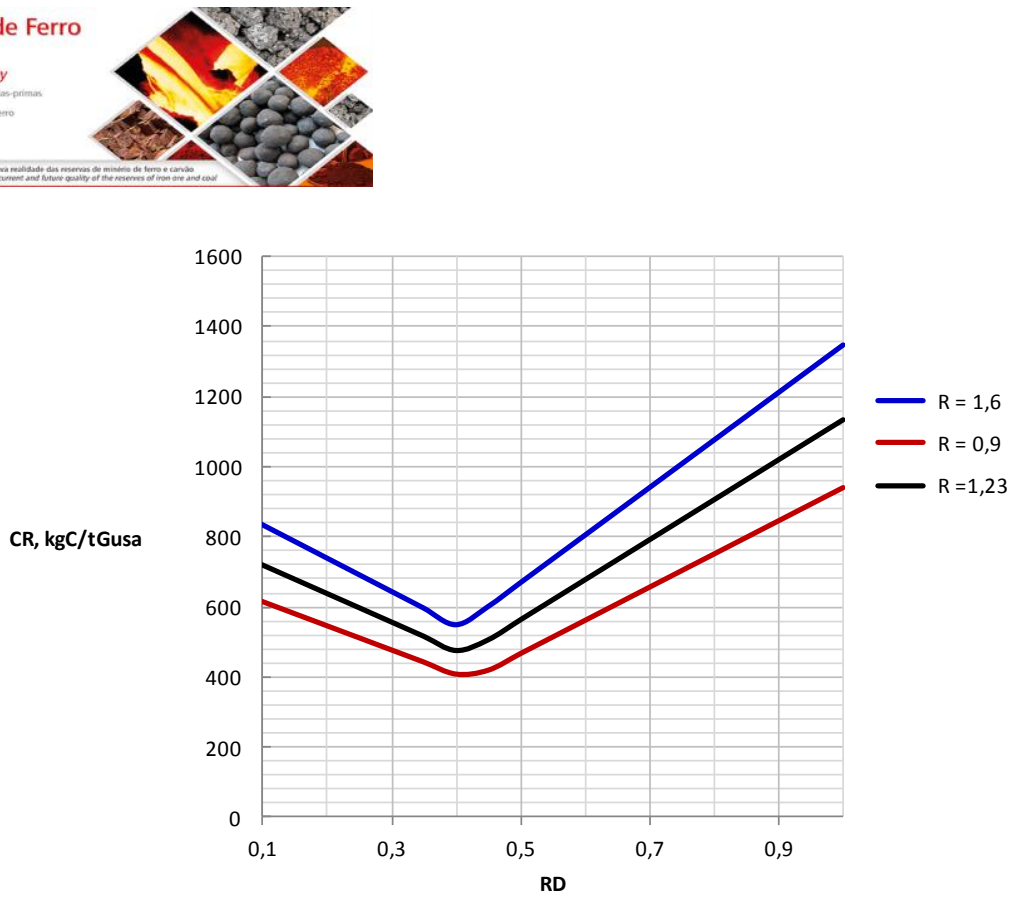

Figura 2. Diagrama CR-RD para $\mathrm{R}$ constante

\section{RESULTADOS}

A fim de exemplificar a aplicação do diagrama em condições reais de um processo de produção de ferro primário, foram selecionadas operações de um alto forno à coque. Duas representando operações diárias regulares e duas outras que apresentaram problemas operacionais de esfriamento do forno, conhecidos como "geladas", num intervalo de cerca de 4 horas.

Nas figuras (3 e 4) são apresentados os cortes CR-RD da superfície de mínimo consumo de carbono, utilizando os seguintes dados básicos de operação:

Operação 1:

"Coke rate" : 464,5 kg coque/t gusa.

$\mathrm{CO} / \mathrm{CO}_{2}$ ] topo: 1,0801

Ômega : :0,0132

Operação 3:

"Coke rate" : $473 \mathrm{~kg}$ coque/t gusa.

$\mathrm{CO} / \mathrm{CO}_{2}$ ] topo: 1,1628

Ômega : 0,0122
Operação 2:

"Coke rate" : 464,5 kg coque/t gusa

$\mathrm{CO} / \mathrm{CO}_{2}$ ] topo: 1,0122

Ômega : : 0,0094

Operação 4:

"Coke rate" : $472,5 \mathrm{~kg}$ coque $/ \mathrm{t}$ gusa

$\mathrm{CO} / \mathrm{CO}_{2}$ ] topo: 0,7557

Ômega : : 0,0272

A figura 3 ilustra as situações do Alto Forno em condições de marchas regulares ( normais ), representadas pelas operações 1 e 2 .
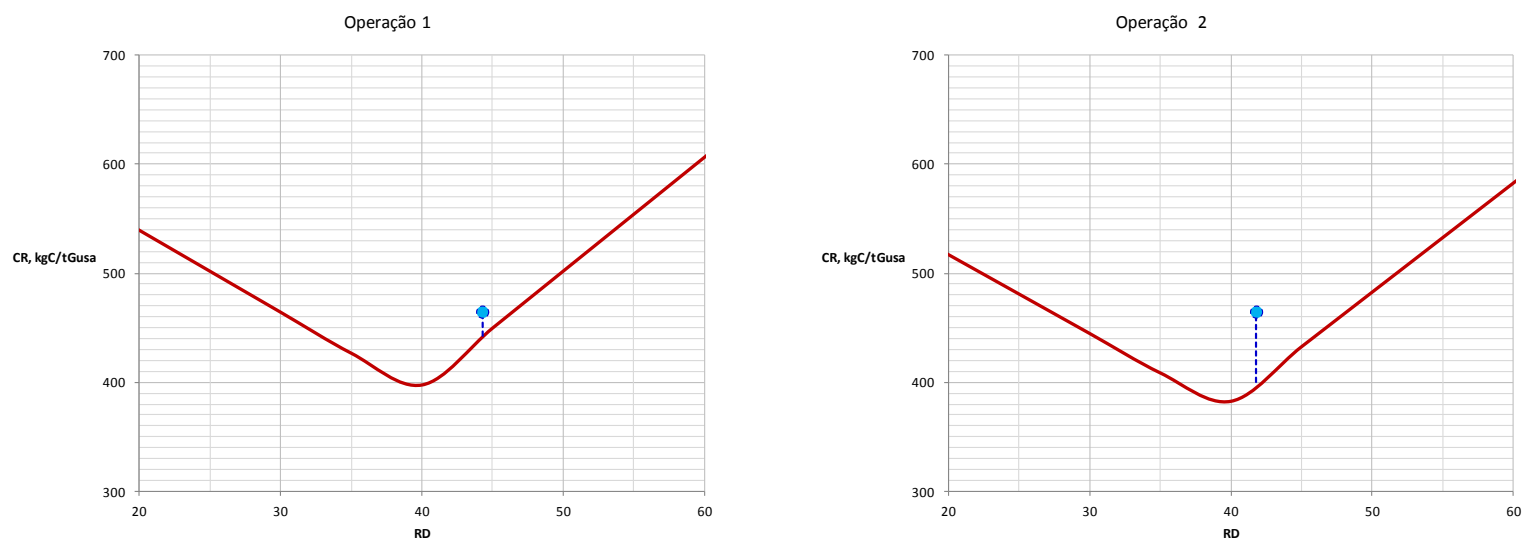

Figura 3. Diagramas CR-RD para operações normais do alto forno (operações 1 e 2), onde estão indicados pontos operacionais reais (em azul) e seu afastamento da fronteira de viabilidade.

* Contribuição técnica ao 44 Seminário de Redução de Minério de Ferro e Matérias-primas, 15ํ Simpósio Brasileiro de Minério de Ferro e 2 Simpósio Brasileiro de Aglomeração de Minério de Ferro, 15 a 18 de setembro de 2014, Belo Horizonte, MG, Brasil. 
A figura 4, por sua vez, mostra condições operacionais anormais (operações 3 e 4), nas quais houve esfriamento do alto forno ("gelada"). Nelas os pontos operacionais se colocaram nitidamente abaixo da linha de consumo mínimo de coque, o que representou condições inviáveis.
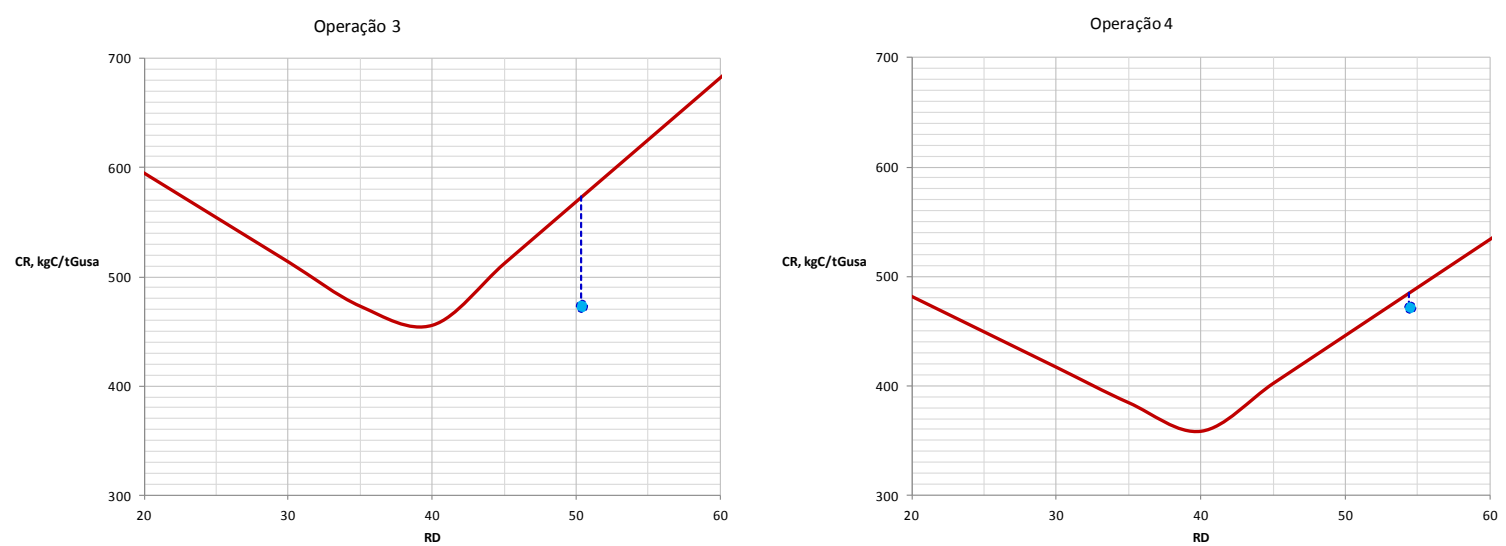

Figura 4. Diagrama CR-RD para operações anormais do alto forno, operação $3(01: 00 \mathrm{~h})$ e operação 4 (05:00 h), onde estão indicados pontos operacionais reais (em azul) e seu afastamento da fronteira de viabilidade.

\section{CONCLUSÕES}

- Foram modeladas as superfícies de mínimo consumo de carbono para os processos de redução dos óxidos de ferro, com base nos equilíbrios das reações e nos balanços de massa e energia;

- O sistema de coordenadas adotado para a geração da superfície de mínimo consumo de carbono, para o caso de altos fornos, foi Carbono Consumido $x$ Relação $\mathrm{CO} / \mathrm{CO}_{2} \times$ \% Redução Direta, tendo em vista a praticidade da função resposta para os planos relativos a $\mathrm{CO} / \mathrm{CO}_{2}$ constantes;

- No caso de operações normais e com esfriamento anormal de um alto forno forno ('gelada'), foi possível identificar com clareza a posição de cada operação em relação `a superfície de mínimo consumo de carbono correspondente, e conduzir às seguintes constatações e confirmações práticas: ponto operacional acima da superfície: operação viável; ponto operacional abaixo da superfície: operação inviável;

- A implementação de acompanhamentos "on line" dos pontos operacionais de um alto forno em relação à superfície de mínimo consumo de carbono, permitirá prever, com antecedência, a tendência da operação estar se encaminhando, ou não, para uma inviabilidade termodinâmica, viabilizando, assim, a tomada antecipada de medidas corretivas.

\section{Agradecimentos}

À CAPES pela bolsa de pós-graduação; ao Grupo de Siderurgia da PUC-Rio/DEMa pelo apoio na pesquisa

* Contribuição técnica ao 44 Seminário de Redução de Minério de Ferro e Matérias-primas, 15은 Simpósio Brasileiro de Minério de Ferro e $2^{\circ}$ Simpósio Brasileiro de Aglomeração de Minério de Ferro, 15 a 18 de setembro de 2014, Belo Horizonte, MG, Brasil. 


\section{REFERÊNCIAS}

1 ABM. Introdução a Siderurgia-coleção de livros metalurgia, Materiais e Mineração. 1a Edição. São Paulo 2011.

2 Wellington AC, Aplicação de um método de Simulação-otimizacão na cadeia produtiva de minérios de ferro, Dissertação de Mestrado. EPUSP, Curso de Pós-Graduação em Engenharia de Sistemas Logísticos, agosto, Setembro de 2007.

3 Bilik J, Kret J, Beer H. Application of The Simulating Mathematical Models for Decreasing of The Blast Furnace Fuel Rate; disponivel em:

http://www.ams.tuke.sk/data/ams_online/1998/number1/mag07.pdf. acessado em Nov 2013.

4 Rizzo EMS. Introdução aos processos Siderúrgicos. São Paulo 2005.

5 Rizzo EMS. Processo de Fabricação de ferro-gusa em Alto-Forno. ABM. São Paulo 2009.

6 Melgaço Jr HA. Aplicação dos métodos gráficos de Rist e Reichardt no controle operacional de Altos Fornos a coque. Dissertação de Mestrado. PUC-RIO, Curso de Pós-Graduação em Engenharia Metalúrgica (orientador: José Carlos D’ABREU). Setembro de 1985.

7 Araújo LA. Manual de siderurgia. $1^{\circ}$ volume. São Paulo: Editora Arte \& Ciencia, 1997.

8 Castro LA. Desenvolvimento De Um Modelo de Controle de Processo Para AltosFornos A Carvão Vegetal, Tese de Doutorado. UFMG, Curso de Pós-Graduação em Engenharia Metalúrgica e de Minas, agosto, 2002.

9 Melo WR, Controle do processo de Redução no Alto-Forno II da Cosipa aplicação do diagrama CRRD. Dissertação de Mestrado. PUC-Rio, Curso de Pós-Graduação em Engenharia Metalúrgica (orientador José Carlos D’Abreu). Fevereiro de 1987.

10 Nakatani TMF, Nakamura F. Theoretical Consideration on Coke Ratio of the Blast Furnace Operation, Steel Science Portal. Novembro de 1965

11 Instituto Aço Brasil, Relatório de sustentabilidade 2013, disponível em: http://www.acobrasil.org.br/site/portugues/sustentabilidade/relatorio.asp acessado em : 10 Maio 2014.

* Contribuição técnica ao 44 Seminário de Redução de Minério de Ferro e Matérias-primas, 15 Simpósio Brasileiro de Minério de Ferro e 2o Simpósio Brasileiro de Aglomeração de Minério de Ferro, 15 a 18 de setembro de 2014, Belo Horizonte, MG, Brasil. 